\title{
Comparative effects of different whole grains and brans on blood lipid: a network meta-analysis
}

\author{
Suocheng Hui ${ }^{1} \cdot$ Kai Liu $^{1,2} \cdot$ Hedong Lang $^{1} \cdot$ Yang Liu $^{1} \cdot$ Xiaolan Wang $^{1} \cdot$ Xiaohui Zhu $^{1} \cdot$ Steve Doucette ${ }^{3} \cdot$ Long Yi $^{1}$. \\ Mantian $\mathrm{Mi}^{1}$
}

Received: 3 April 2018 / Accepted: 18 September 2018 / Published online: 22 September 2018

(c) The Author(s) 2018

\begin{abstract}
Purpose The comparative effects of different whole grains and brans on blood lipid are still not totally elucidated. We aimed to estimate and rank the effects of different whole grains and brans on the control of blood lipid.

Methods We performed a strategic literature search of PubMed, EMBASE and the Cochrane Library for relevant trials. Both pairwise meta-analyses and network meta-analyses were conducted to compare and rank the intervention strategies of whole grains and brans for the control of total cholesterol (TC), LDL cholesterol (LDL-C), HDL cholesterol (HDL-C), and triglycerides (TG).

Results Fifty-five eligible trials with a total of 3900 participants were included. Cumulative ranking analyses showed that oat bran was the most effective intervention strategy for TC and LDL-C improvements, with significant decreases of $-0.35 \mathrm{mmol} / \mathrm{L}(95 \% \mathrm{CI}-0.47,-0.23 \mathrm{mmol} / \mathrm{L})$ and $-0.32 \mathrm{mmol} / \mathrm{L}(95 \% \mathrm{CI}-0.44,-0.19 \mathrm{mmol} / \mathrm{L}) \mathrm{in} \mathrm{TC}$ and LDL-C compared with control, respectively. In comparison with control, oat was associated with significant reductions in TC by $-0.26 \mathrm{mmol} / \mathrm{L}(95 \% \mathrm{CI}-0.36,-0.15 \mathrm{mmol} / \mathrm{L})$ and LDL-C by $-0.17 \mathrm{mmol} / \mathrm{L}(95 \% \mathrm{CI}-0.28,-0.07 \mathrm{mmol} / \mathrm{L})$, which was ranked as the second best treatment. Barley, brown rice, wheat and wheat bran were shown to be ineffective in improving blood lipid compared with control.

Conclusions This network meta-analysis suggests that oat bran and oat are ranked higher than any other treatments for the regulations of TC and LDL-C, indicating that increasing oat sources of whole grain may be recommended for lipid control.
\end{abstract}

Keywords Whole grain $\cdot$ Bran $\cdot$ Blood lipid $\cdot$ Network meta-analysis

\section{Introduction}

Suocheng Hui and Kai Liu contributed equally to this work.

Electronic supplementary material The online version of this article (https://doi.org/10.1007/s00394-018-1827-6) contains supplementary material, which is available to authorized users.

Mantian Mi

mi_mt2009@hotmail.com

1 Research Center for Nutrition and Food Safety, Institute of Military Preventive Medicine, Third Military Medical University, Chongqing Key Laboratory of Nutrition and Food Safety, Chongqing Medical Nutrition Research Center, Chongqing 400038, People's Republic of China

2 Department of Health Supervision, Center for Disease Control and Prevention of Shenyang Joint Logistic Support Center, Shenyang 110034, People's Republic of China

3 Department of Community Health and Epidemiology, Dalhousie University, Halifax, Nova Scotia, Canada
According to the latest estimates, $31 \%$ of all global deaths (17.7 million) are due to cardiovascular diseases (CVDs) [1]. The management of CVDs is costly and prolonged, which has brought heavy burden to the public health [2, 3]. Several previous studies demonstrated that effectively control of lipid metabolism inhibited the development and progression of CVDs [4, 5]. In addition, it has been suggested that $1 \%$ decreases in total cholesterol (TC) and LDL cholesterol (LDL-C) can reduce the risk of CVDs by $3 \%$ and $1 \%$, respectively [6]. Recently, dietary intervention strategies have received increasing attention in the prevention and treatment of CVDs because they may have less adverse effects and are more cost-effective compared with the lipid lowering drugs $[7,8]$.

Among various dietary adjustment strategies, increasing whole grains and brans intake has been widely 
investigated in the area of blood lipid control $[9,10]$. Common whole grains and brans used in the previous clinical trials included oat, wheat, brown rice, barley, oat bran and wheat bran $[11,12]$. However, these different intervention strategies have yielded variable results and which type is superior to others in lipid control are still inconclusive.

There is no individual RCT investigating the comparative effects of different whole grains and brans on blood lipid, which might because it is rather costly to conduct an individual trial with more than three arms [13]. Additionally, the traditional meta-analysis methods do not allow different comparators to be compared simultaneously and thus they have limited ability to rank the relative effectiveness of different interventions [13, 14]. However, network meta-analysis incorporates both direct and indirect comparisons of different intervention strategies, including those that have never been compared directly in head-tohead trials, which can answer questions more broadly than pairwise meta-analysis [15]. Therefore, we conducted a network meta-analysis to adequately assess and rank the comparative effects of different whole grains and brans on the control of blood lipid.

\section{Materials and methods}

\section{Literature search}

The study was conducted according to the prespecified study protocol and preferred reporting items for systematic reviews and meta-analyses (PRISMA) extension statement for reporting of network meta-analyses of health care interventions [16]. PubMed (updated to July 2018; http:// www.ncbi.nlm.nih.gov/pubmed/), Embase (updated to July 2018; http://www.embase.com/search/advanced/) and the Cochrane Library (updated to July 2018; http://www.cochr ane.org/) were searched for human studies. Following terms were searched in all fields: whole grain, wholegrain, whole meal, whole wheat, wheat, rice, brown rice, wild rice, maize, oat, barley, corn, rye, millet, sorghum, triticale, canary seed, amaranth, buckwheat or quinoa which were paired with the following words: lipid profile, lipid distribution, blood lipid, cholesterol, total cholesterol, TC, low density lipoprotein, LDL, LDL-C, high density lipoprotein, HDL, HDL-C, triglyceride, triacylglycerol, triglyceride, TG, TAG or lipoprotein. In addition, to further identify eligible trials, we hand-searched reference lists in the reviews and included studies. The full search strategy was described in Supplemental materials and methods. The literature search and study selection were conducted by two independent reviewers in parallel (SC-H and K-L), and any discrepancies were resolved by a third investigator (MT-M).

\section{Study selection}

We selected studies that met the following criteria: (1) treatment duration lasted more than 2 weeks; (2) the studies enrolled apparently healthy or high-risk CVDs population (subjects with known dyslipidemia, hyperglycemia, hypertension, overweight or obesity, or a combination of these factors) and not diagnosed with CVDs [17, 18]; (3) the baseline and post-intervention values or change scores for TC, LDL-C, HDL-C, or TG with their standard deviations (SDs), standard errors (SEs) or 95\% CIs were available for each group in the study; (4) participants received the intervention of barley, brown rice, oat, oat bran, rye, rye bran, wheat, wheat bran alone or control (refined-grain diets or products); (5) the trial compared 2 or more different intervention strategies; (6) the treatment products were not given as multi-components and the effects of whole grains and brans could be distinguished.

\section{Risk of bias and quality of evidence}

The risk of bias was assessed using the Cochrane Risk of Bias assessment tool. The assessment items included adequacy of sequence generation, allocation concealment, blinding, blinding of outcome assessments, incomplete outcome data, selective reporting, and other biases [19]. Additionally, the quality of evidence was assessed using the Grading of Recommendations Assessment, Development and Evaluation (GRADE) framework, which characterizes the evidence on the basis of the study limitations, imprecision, inconsistency, indirectness, and publication bias [20]. Two researchers (SC-H and K-L) independently reviewed the studies and judged the risk of bias and quality of evidence. Any discrepancies were resolved by consensus and arbitration by a third investigator (MT-M).

\section{Data extraction}

We reviewed the included articles and the following items were extracted: (1) study characteristics including information of authors, publication year, region, sample size, study design, treatment duration, treatment products and dietary fiber content of intervention products; (2) population information on age and body mass index (BMI); (3) change scores (primary values) or baseline and postintervention values in TC, LDL-C, HDL-C and TG; (4) all values were converted to $\mathrm{mmol} / \mathrm{L}$ using the conversion factors $1 \mathrm{mg} / \mathrm{dL}=0.02586 \mathrm{mmol} / \mathrm{L}$ for TC, LDL-C, HDL-C, and $1 \mathrm{mg} / \mathrm{dL}=0.01129 \mathrm{mmol} / \mathrm{L}$ for TG [21]. For crossover design trials, we extracted the data of two phases [19]. In parallel design studies, all treatment outcomes at 
different visits were extracted and used to estimate the intervention effects.

\section{Statistical analysis}

We performed the pairwise meta-analysis using the randomeffects model. The outcomes of treatments were estimated using mean differences (MDs). The $I^{2}$ statistic and $P$ value were calculated to identify the heterogeneity among the included studies [22]. In addition, the Egger's test was used to detect the small-study effects.

We excluded percentage changes in mean and SD values when we extracted data for the meta-analysis. If SD values were not reported in the studies, we calculated them from SEs, 95\% CIs, $P$ values, or $t$ statistics. Besides, change-frombaseline SD values were calculated by assuming a correlation coefficient of 0.5 [23].

Frequentist model network meta-analyses were conducted to estimate the comparative effects of different whole grains and brans on the control of blood lipid if at least five treatments arms were available across the studies [24]. Ranking probabilities of treatments were evaluated using surface under the cumulative ranking curve (SUCRA) and mean ranks. The SUCRA accounts both for the variance and the location of all relative treatment effects. The larger the SUCRA value, the better the rank of the treatment $(0 \%=$ worst; $100 \%$ = best) $[25,26]$.

Transitivity is the fundamental premise underlying network meta-analysis [13, 14, 27]. We evaluated whether the transitivity assumption is valid by assessing the inconsistency between direct and indirect evidence. We assessed the local inconsistency using the loop-specific approach and node-splitting method [28, 29]. To assess the evidence of inconsistency in the entire network, we used the design-bytreatment model [30]. We used the comparison-adjusted funnel plot to visually assess the evidence for publication bias in the network [25], and the comparisons were presented as treatment alphabetically earlier versus later treatment.

To assess the robustness of the findings, we performed sensitivity analyses by fitting the inconsistency model as described by White [31, 32]. To explore the influence of study design on the results of network meta-analysis, we performed sensitivity analyses by only including the studies with a parallel design or removing the cluster crossover design trials. To explore the effect of treatment duration on the overall results of network meta-analysis, we conducted sensitivity analyses by excluding studies with the duration less than 3, 4 weeks or more than 12 weeks. In addition, we also conducted sensitivity analyses based on the studies that only included dyslipidemic participants, or low risk of bias.

We did the pairwise meta-analysis using meta package for R software and network meta-analysis with the method of multivariate meta-analysis in Stata version 14.0 using the mvmeta command and Stata routines described elsewhere [25, 31-34].

\section{Results}

\section{Literature search}

The detailed process of search strategy for this meta-analysis is shown in Fig. 1. A total of 6838 articles were identified in the initial search, and 6589 were excluded after reviewing the titles and abstracts. Among the excluded reports, 4726 were excluded because they were not relevant to the network meta-analysis and 1863 were excluded because they were duplicates. Therefore, 249 articles were remained for further detailed examinations. Among these 249 articles, an additional 194 were then excluded for the following reasons: 120 were excluded because they used multiple components,

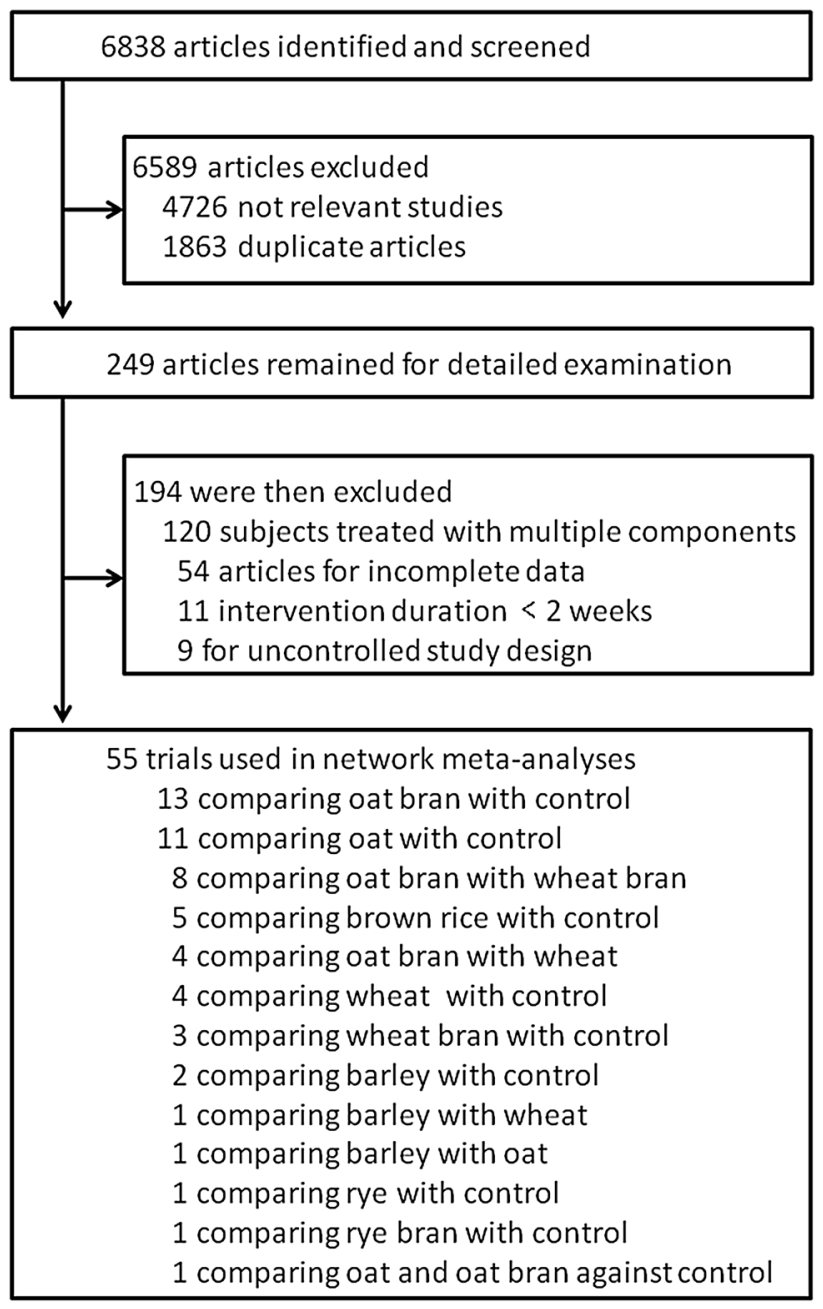

Fig. 1 Flow diagram showing the number of citations retrieved in individual searches of articles included in the review 
54 were discarded because they had incomplete data, 11 were excluded because the treatment duration lasted less than 2 weeks, and 9 were ruled out because they used an uncontrolled study design. Thus, 55 studies were ultimately selected in this network meta-analysis.

\section{Study characteristics}

A summary of study characteristics was presented in Supplemental Table 1. Fifty-five studies comprising a total of 3900 participants were included in the network meta-analysis. The total number of subjects included in each study ranged from 8 to 367 . The BMI of the participants ranged from 19.2 to $30.9 \mathrm{~kg} / \mathrm{m}^{2}$ (median $26.8 \mathrm{~kg} / \mathrm{m}^{2}$ ). The age of the participants ranged from 20.4 to 63.0 years (median 51.0 years). Most of the studies ( 35 of 55) used the parallel design and the remained 20 studies used the crossover design. The intervention duration varied from 2 weeks to 12 months (median 6 weeks). Funding sources for all included studies were presented in Supplemental table 2.

\section{Risk of bias and quality of evidence}

The risk of bias of the studies included in this network metaanalysis was generally low or unclear. Overall, 2 studies had a high-risk of bias for random sequence generation, 8 had unclear risk of bias on allocation concealment. 23 studies had a low risk of bias on blinding and 20 were in low risk of bias on blinding of outcome assessment. Most articles were in low risk of incomplete outcome data, selective reporting and other biases (Supplemental Figs. 1 and 2). The quality of evidence for all outcomes was rated as moderate or low for most comparisons. More details of the quality of evidence were presented in Supplemental tables 16, 30, 44 and 58.

\section{Pairwise meta-analysis}

The pairwise meta-analysis showed that oat significantly reduced TC $(-0.30 \mathrm{mmol} / \mathrm{L} ; 95 \% \mathrm{CI}-0.42$, $-0.19 \mathrm{mmol} / \mathrm{L})$ and LDL-C $(-0.16 \mathrm{mmol} / \mathrm{L} ; 95 \% \mathrm{CI}-0.27$, $-0.05 \mathrm{mmol} / \mathrm{L}$ ) compared with control. Compared with control, significant decreases in TC $(-0.27 \mathrm{mmol} / \mathrm{L} ; 95 \% \mathrm{CI}$ $-0.43,-0.12 \mathrm{mmol} / \mathrm{L})$ and LDL-C $(-0.34 \mathrm{mmol} / \mathrm{L} ; 95 \%$ CI $-0.63,-0.05 \mathrm{mmol} / \mathrm{L}$ ) were observed in the oat bran group (Supplemental Figs. 3 and 5). Compared with control, no remarkable improvements of HDL-C and TG were shown after the treatment of whole grains and brans (Supplemental Figs. 7 and 9). Additionally, most comparisons in all outcomes did not showed significant heterogeneity. Detailed results were summarized in Supplemental Tables 3, 17,31 and 45.

\section{Network meta-analysis}

We did not conduct network meta-analysis for rye and rye bran because there was only one study available for analysis, respectively. The networks of eligible comparisons for TC, LDL-C, HDL-C and TG are shown in Fig. 2a-d, respectively. The network meta-analysis suggested that oat bran had the greatest likelihood of being the most effective treatment for TC, followed by oat (Fig. 3 and Supplemental Table 7). Consistent with the results of
Fig. 2 Network plots of eligible comparisons for different whole grains and brans for TC (a), LDL-C (b), HDL-C (c) and TG (d). Lines connect the interventions that have been studied in head-to-head (direct) comparisons in the eligible studies. The sizes of the nodes are weighted according to the number of trials that study the intervention, and the thickness line corresponds to the number of trials that assess direct comparisons between different interventions
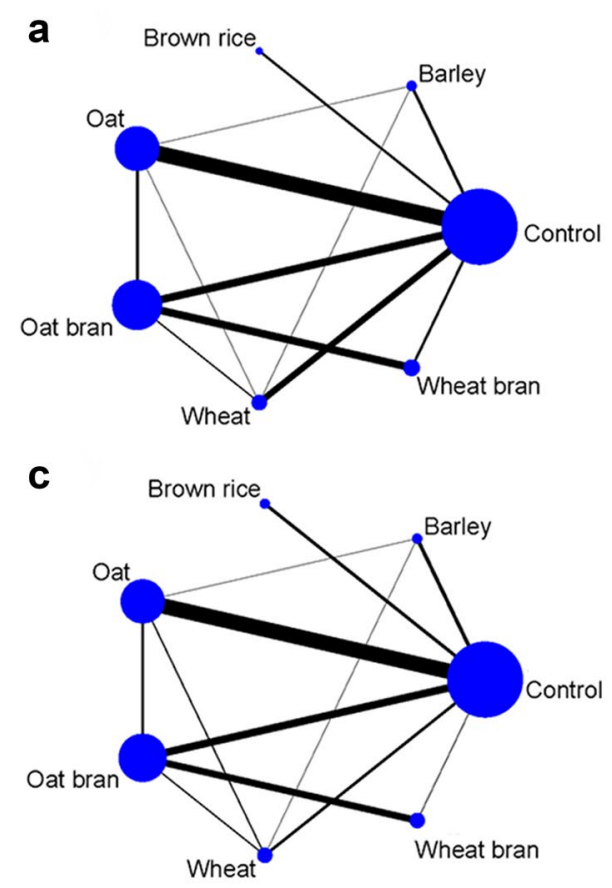

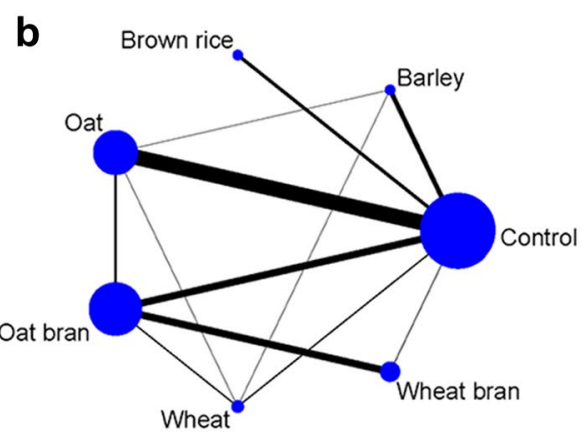

d

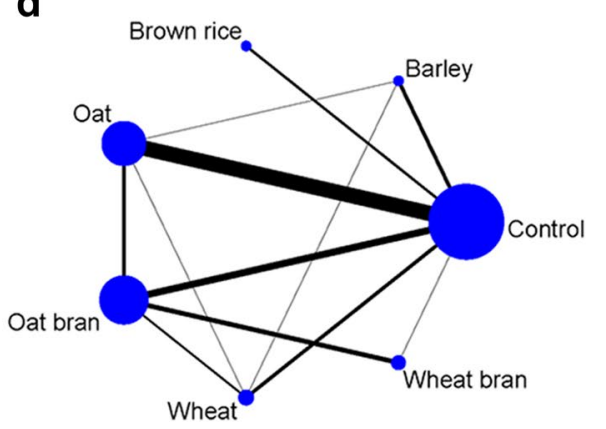




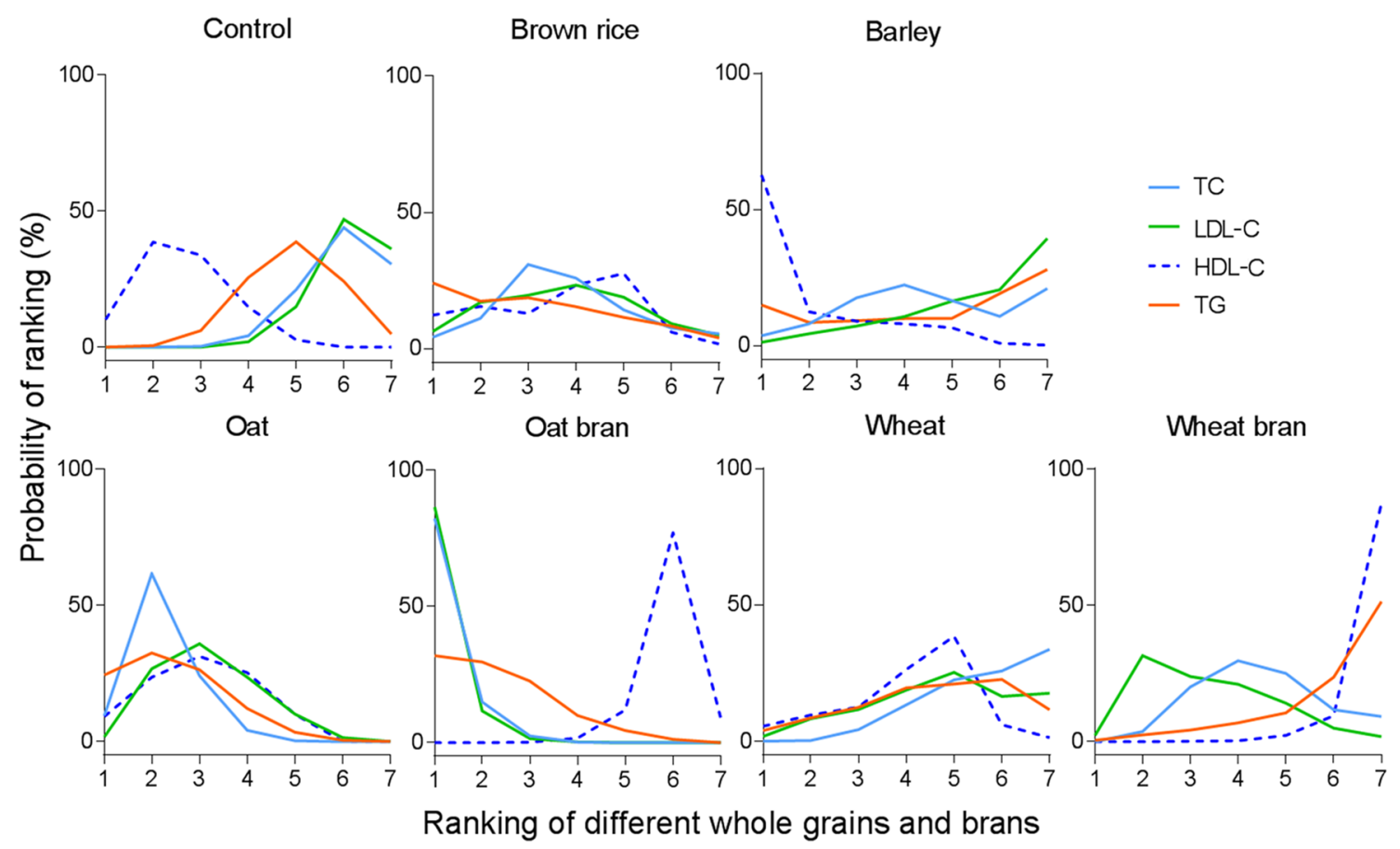

Fig. 3 Ranking results of different whole grains and brans on the control of blood lipid. The $x$-axis represents the ranking of interventions which are ranked in numerical order, with the first representing the

pairwise meta-analysis, compared with control, oat and oat bran significantly decreased TC by $-0.26 \mathrm{mmol} / \mathrm{L}(95 \%$ $\mathrm{CI}-0.36,-0.15 \mathrm{mmol} / \mathrm{L})$ and $-0.35 \mathrm{mmol} / \mathrm{L}(95 \% \mathrm{CI}$ $-0.47,-0.23 \mathrm{mmol} / \mathrm{L})$, respectively. In addition, oat bran and oat were superior to wheat in reducing TC. Barley, brown rice, wheat and wheat bran showed insignificant effects on TC. Detailed results of network meta-analysis on TC are shown in Supplemental Table 6.

As presented in Fig. 3 and Supplemental Table 21, results of the network meta-analysis on LDL-C demonstrated that oat bran was the highest ranked treatment strategy for the reduction of LDL-C, followed by oat. Detailed results were available in Supplemental Table 20. Compared with control, oat and oat bran significant reduced LDL-C by $-0.17 \mathrm{mmol} / \mathrm{L}$ ( $95 \% \mathrm{CI}-0.28,-0.07 \mathrm{mmol} / \mathrm{L}$ ) and $-0.32 \mathrm{mmol} / \mathrm{L}(95 \% \mathrm{CI}-0.44,-0.19 \mathrm{mmol} / \mathrm{L})$, respectively. Besides, oat bran was more effective than brown rice and wheat for LDL-C reductions. The same to the results of TC, increasing the consumption of barley, brown rice, wheat or wheat bran was not associated with reduction of LDL-C.

Estimated effects of different whole grains and brans on HDL-C were presented in Supplemental Table 34. Based on the ranking results of HDL-C, brown rice appeared to be the most effective intervention, and oat was ranked after brown rice (Fig. 3 and Supplemental Table 35). However, compared best. The $y$-axis represents the probability of each ranking. Each line indicates a type of intervention strategy

with control, consumption of different whole grains and brans showed no pronounced beneficial effects on HDL-C.

Consistent with the results of TC and LDL-C, the ranking analysis on TG showed that oat bran had the highest possibility to be the best intervention strategy, followed by oat (Fig. 3 and Supplemental Table 49). However, the results of network meta-analysis suggested that of oat bran and oat did not significantly affect the concentration of TG compared with control. In addition, oat bran exerted greater decreased effects on TG when compared with wheat bran $(-0.11 \mathrm{mmol} / \mathrm{L}, 95 \% \mathrm{CI}:-0.21,-0.01 \mathrm{mmol} / \mathrm{L}$; Supplemental Table 48).

Contributions of direct evidence to the network analysis are reported in Supplemental Tables 4, 18, 32 and 46. The analysis of design-by-treatment model did not identify any significant global inconsistency for TC $\left(\tau^{2}=0.017\right.$, $P=0.81)$, LDL-C $\left(\tau^{2}=0.018, P=0.85\right)$, HDL-C $\left(\tau^{2}=0.005\right.$, $P=0.17)$ and TG $\left(\tau^{2}=0.005, P=0.99\right)$. Overall, we did not observed any of inconsistencies between evidence derived from direct and indirect comparisons using the node-splitting method. For details of the assessments of inconsistency see Supplemental Tables 5, 19, 33 and 47. Loop-specific analyses indicated that treatment effects estimated from direct and indirect evidence in general did not show significant statistical inconsistencies. Details of the assessments of inconsistency for TC, LDL-C, HDL-C and TG are shown in Supplemental Figs. 4, 6, 8 and 10. The above results of test 


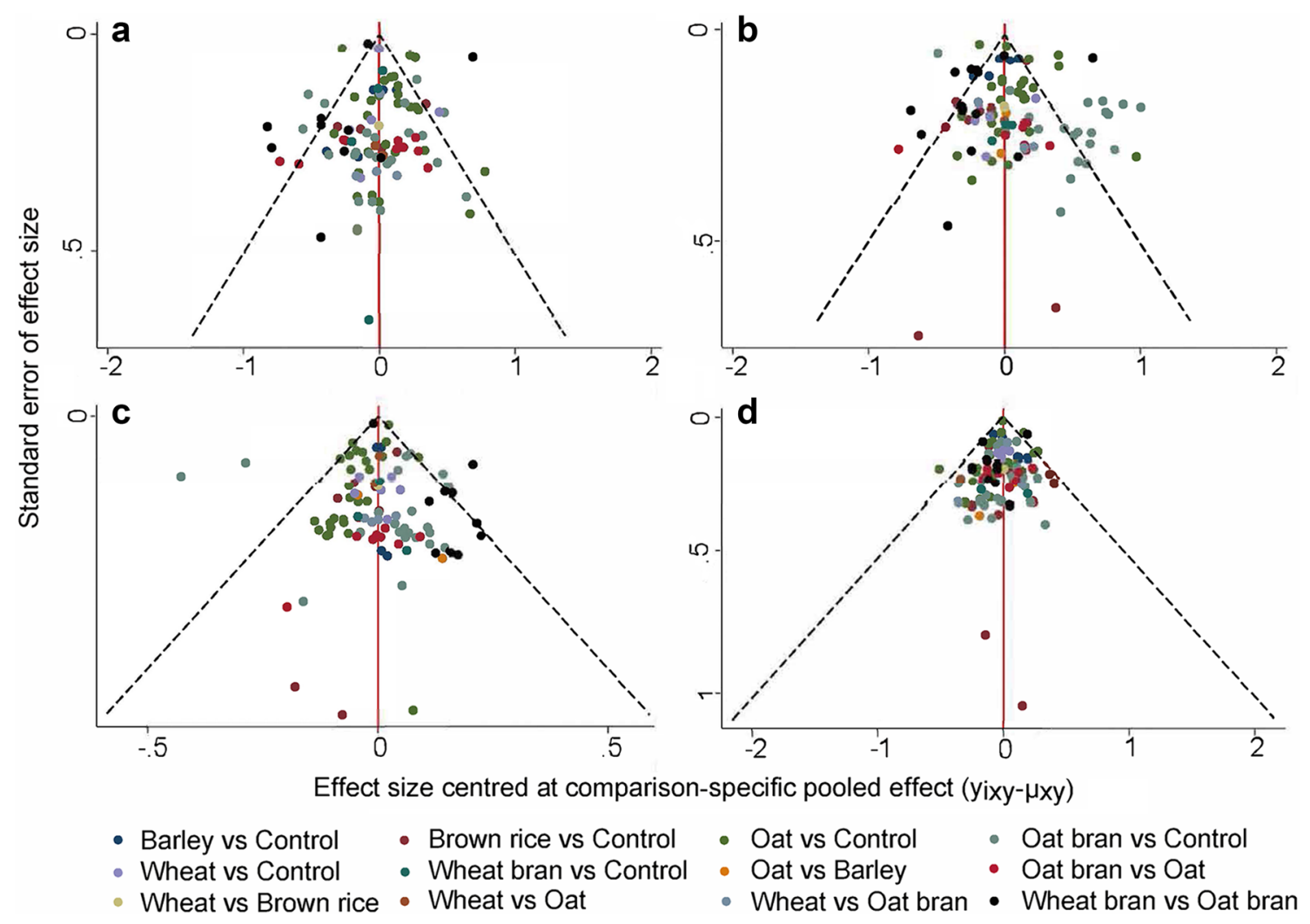

Fig. 4 Comparison-adjusted funnel plots for TC (a), LDL-C (b), HDL-C (c) and TG (d). The red line represents the null hypothesis that the study-specific effect sizes do not differ from the respective comparison-specific pooled effect estimates. The two black dashed

for inconsistency supported the assumption of transitivity in this network meta-analysis. Finally, the comparison-adjusted funnel plots of the network meta-analysis for TC, LDL-C, HDL-C and TG did not show any significant publication bias (Fig. 4a-d).

The sensitivity analyses using inconsistency model showed similar results with main analyses. In addition, the sensitivity analyses only including the participants with dyslipidemia showed that oat bran was more effective than oat on the reduction of LDL-C. Moreover, the overall outcomes for all variables were not significantly changed when we only including the studies with a parallel design or removing the cluster crossover design trials. Finally, sensitivity analyses based on treatment duration and risk of bias of trials were similar with the main results. Detailed results of the sensitivity analyses are reported in the Supplemental Tables 8-15, 22-29, 36-43 and 50-57. lines represent a 95\% CI for the difference between study-specific effect sizes and comparison-specific summary estimates. Different colors correspond to different comparisons

\section{Discussion}

To our knowledge, this is the first network meta-analysis investigating the comparative effects of different whole grains and brans on blood lipid. A particular strength of network meta-analysis is that one can obtain the relative effect estimations among different interventions by incorporating direct and indirect evidence [14, 15]. In this network meta-analysis, we found that oat bran might be the most effective intervention strategy for TC and LDL-C reductions, while oat ranked the second based on cumulative ranking analysis. Although estimates of effects size varied among different interventions, oat bran and oat were more or no less effective compared with any other types whole grains and brans. Conversely, barley, brown rice, wheat, and wheat bran showed insignificant effects on blood lipid. As suggested by these findings, increasing consumption of oat sources of whole grain might be necessary to dietary adjustment for lipid control.

Evidence about comparative effects of different whole grains and brans on the control of blood lipid is still limited. In this study, we found that oat bran was ranked as the best intervention strategy for TC and LDL-C regulations, and oat 
was ranked as the second position. Additionally, we demonstrated that oat bran and oat had more or potential favorable effects on lipid control compared with other interventions. The advantage of oat bran and oat in lipid control might be attributed to their high proportion of $\beta$-glucan and the mechanism underlying may involve the following reasons: first, $\beta$-glucan can act as a physical barrier by inhibiting the absorption of bile acids which is associated with the synthesis and dissociation of cholesterol [35]; second, $\beta$-glucan can decrease the enterohepatic circulation of bile acids and increase the excretion of fecal bile acids by binding with bile acids in small intestine [36, 37]; third, $\beta$-glucan can lower insulin concentrations through suppressing the absorption of carbohydrate and in turn decrease the synthesis of cholesterol [38]. In addition, $\beta$-glucan can improve the blood lipid by modifying the composition of gut microbiota such as increasing the microbial diversity, abundance of the genus Bacteroidaceae, and the ratio of Bacteroidetes/Firmicutes [39-41].

Although we did not find significant differences between oat bran and oat on the control of blood lipid, the sensitivity analysis based on the studies that only included dyslipidemic participants showed that oat bran was more effective than oat on the reduction of LDL-C. The possibility might be that bran is the major and direct source of fiber, minerals, magnesium and phytonutrients [12, 42], which are the primary nutrients responsible for health benefits in whole grains, but with low calorie. Thus, increasing the quantity of whole grain brans alone might provide enough nutrients for health benefits, but without excessive calories intake [43].

This network meta-analysis also has some limitations. First, to address the independent effect of the different whole grains and brans on blood lipid, the studies used mixed whole grains and brans were excluded. The network metaanalysis found that intervention with barley, brown rice, wheat, and wheat bran alone could not markedly improve the blood lipid which might be due to the limited number of head-to-head trials. Although these results are consistent with previous traditional meta-analysis [11], more related high-quality trials with large-scale and well-controlled design are needed to provide clearer answers and more evidence. Second, our network meta-analysis suggested TC and LDL-C were significantly decreased after oat bran and oat interventions. However, it is difficult to evaluate the association of the reductions of TC and LDL-C found in this study in terms of CVDs risk reduction because most of the study durations were less than 1 year and data for relative risk evaluation were not available in the included studies. Third, evidence in this network meta-analysis largely originated from East Asia, North America, and Europe, with fewer from regions such as Southeast Asia, South America, and Africa. Thus, to better understand the effects of whole grains and brans on lipids control, further studies from these regions are required. Fourth, most studies included in our network meta-analysis were placebo controlled trials, the number of head-to-head trials which comparing different active treatments directly is still limited. Future direct comparison trials are needed to further evaluate and confirm our findings. Furthermore, publication bias and heterogeneity are the inevitable problems in the meta-analysis. However, the Egger's tests and comparison-adjusted funnel plots suggested that no significant publication bias exists in this study. In addition, the results of sensitivity analyses based on study design, intervention duration, baseline lipid level and risk of bias were consistent to the main results. Moreover, the control interventions, as the common comparator, across the included studies were similar and the results of test for inconsistency suggested the assumption of transitivity was valid.

Overall, the present network meta-analysis provided comprehensive evidence about the comparative effects of different whole grains and brans on the control of blood lipid. Both oat bran and oat showed significant lowering effects on TC and LDL-C. In addition, oat bran might be the most optimal strategy for the control of TC and LDL-C, while oat was ranked as the second. The findings suggest that increasing oat sources of whole grain may be beneficial for lipid management.

\section{Compliance with ethical standards}

Conflict of interest None of the authors declared a conflict of interest.

Open Access This article is distributed under the terms of the Creative Commons Attribution 4.0 International License (http://creativeco mmons.org/licenses/by/4.0/), which permits unrestricted use, distribution, and reproduction in any medium, provided you give appropriate credit to the original author(s) and the source, provide a link to the Creative Commons license, and indicate if changes were made.

\section{References}

1. World Health Organisation (2017) New initiative launched to tackle cardiovascular disease, the world's number one killer. http://www.who.int/cardiovascular_diseases/en/. Accessed 23 Mar 2018

2. GBD 2013 Mortality and Causes of Death Collaborators (2015) Global, regional, and national age-sex specific all-cause and cause-specific mortality for 240 causes of death, 1990-2013: a systematic analysis for the Global Burden of Disease Study 2013. Lancet 385:117-171. https://doi.org/10.1016/s0140 $-6736(14) 61682-2$

3. Murray CJ, Lopez AD (1997) Alternative projections of mortality and disability by cause 1990-2020: global burden of disease study. Lancet 349:1498-1504. https://doi.org/10.1016/s0140 $-6736(96) 07492-2$

4. Lloyd-Jones D, Adams RJ, Brown TM, Carnethon M, Dai S, De Simone G, Ferguson TB, Ford E, Furie K, Gillespie C, Go A, 
Greenlund K, Haase N, Hailpern S, Ho PM, Howard V, Kissela B, Kittner S, Lackland D, Lisabeth L, Marelli A, McDermott MM, Meigs J, Mozaffarian D, Mussolino M, Nichol G, Roger VL, Rosamond W, Sacco R, Sorlie P, Roger VL, Thom T, WasserthielSmoller S, Wong ND, Wylie-Rosett J (2010) Heart disease and stroke statistics-2010 update: a report from the American Heart Association. Circulation 121:e46-e215. https://doi.org/10.1161/ circulationaha.109.192667

5. Wolever TM, Tosh SM, Gibbs AL, Brand-Miller J, Duncan AM, Hart V, Lamarche B, Thomson BA, Duss R, Wood PJ (2010) Physicochemical properties of oat beta-glucan influence its ability to reduce serum LDL cholesterol in humans: a randomized clinical trial. Am J Clin Nutr 92:723-732. https://doi.org/10.3945/ ajcn.2010.29174

6. Expert Panel on Detection, Evaluation, Treatment of High Blood Cholesterol in Adults (2002) Third report of the National Cholesterol Education Program (NCEP) Expert Panel on Detection, Evaluation, and Treatment of High Blood Cholesterol in Adults (Adult Treatment Panel III) final report. Circulation 106:3143-3421

7. Zhao CN, Meng X, Li Y, Li S, Liu Q, Tang GY, Li HB (2017) Fruits for prevention and treatment of cardiovascular diseases. Nutrients. https://doi.org/10.3390/nu9060598

8. Backes JM, Ruisinger JF, Gibson CA, Moriarty PM (2017) Statin-associated muscle symptoms-managing the highly intolerant. J Clin Lipidol 11:24-33. https://doi.org/10.1016/j. jacl.2017.01.006

9. Chen GC, Tong X, Xu JY, Han SF, Wan ZX, Qin JB, Qin LQ (2016) Whole-grain intake and total, cardiovascular, and cancer mortality: a systematic review and meta-analysis of prospective studies. Am J Clin Nutr 104:164-172. https://doi.org/10.3945/ ajcn.115.122432

10. Zong G, Gao A, Hu FB, Sun Q (2016) Whole grain intake and mortality from all causes, cardiovascular disease, and cancer: a meta-analysis of prospective cohort studies. Circulation 133:2370-2380. https://doi.org/10.1161/circulationaha.115.02110 1

11. Hollaender PL, Ross AB (2015) Whole-grain and blood lipid changes in apparently healthy adults: a systematic review and meta-analysis of randomized controlled studies. 102:556-572. https://doi.org/10.3945/ajen.115.109165

12. Slavin JL, Martini MC, Jacobs DR Jr, Marquart L (1999) Plausible mechanisms for the protectiveness of whole grains. Am J Clin Nutr 70:459s-463s

13. Salanti G (2012) Indirect and mixed-treatment comparison, network, or multiple-treatments meta-analysis: many names, many benefits, many concerns for the next generation evidence synthesis tool. Res synth Methods 3:80-97. https://doi.org/10.1002/ jrsm. 1037

14. Cipriani A, Higgins JP, Geddes JR, Salanti G (2013) Conceptual and technical challenges in network meta-analysis. Ann Intern Med 159:130-137. https://doi.org/10.7326/0003-4819-159-2201307160-00008

15. Samara MT, Dold M, Gianatsi M, Nikolakopoulou A, Helfer B, Salanti G, Leucht S (2016) Efficacy, acceptability, and tolerability of antipsychotics in treatment-resistant schizophrenia: a network meta-analysis. JAMA Psychiatry 73:199-210. https://doi. org/10.1001/jamapsychiatry.2015.2955

16. Hutton B, Salanti G, Caldwell DM, Chaimani A, Schmid CH, Cameron C, Ioannidis JP, Straus S, Thorlund K, Jansen JP, Mulrow C, Catala-Lopez F, Gotzsche PC, Dickersin K, Boutron I, Altman DG, Moher D (2015) The PRISMA extension statement for reporting of systematic reviews incorporating network metaanalyses of health care interventions: checklist and explanations. Ann Intern Med 162:777-784. https://doi.org/10.7326/m14-2385
17. Lu Y, Wang P, Zhou T, Lu J, Spatz ES, Nasir K, Jiang L, Krumholz HM (2018) Comparison of prevalence, awareness, treatment, and control of cardiovascular risk factors in China and the United States. J Am Heart Assoc. https://doi.org/10.1161/jaha.117.00746 2

18. Lind L, Sundstrom J, Arnlov J, Lampa E (2018) Impact of aging on the strength of cardiovascular risk factors: a longitudinal study over 40 years. J Am Heart Assoc. https://doi.org/10.1161/ jaha.117.007061

19. Higgins JPT, Green S (eds) (2011) Cochrane handbook for systematic reviews of interventions version 5.1.0 (updated March 2011). The Cochrane Collaboration, Available from http://www. handbook.cochrane.org

20. Salanti G, Del Giovane C, Chaimani A, Caldwell DM, Higgins JP (2014) Evaluating the quality of evidence from a network meta-analysis. PLoS One 9:e99682. https://doi.org/10.1371/journ al.pone.0099682

21. Liu K, Xing A, Chen K, Wang B, Zhou R, Chen S, Xu H, Mi M (2013) Effect of fruit juice on cholesterol and blood pressure in adults: a meta-analysis of 19 randomized controlled trials. PLoS One 8:e61420. https://doi.org/10.1371/journal.pone.0061420

22. Higgins JP, Thompson SG, Deeks JJ, Altman DG (2003) Measuring inconsistency in meta-analyses. BMJ 327:557-560. https:// doi.org/10.1136/bmj.327.7414.557

23. Follmann D, Elliott P, Suh I, Cutler J (1992) Variance imputation for overviews of clinical trials with continuous response. J Clin Epidemiol 45:769-773

24. Tricco AC, Ashoor HM, Antony J, Beyene J, Veroniki AA, Isaranuwatchai $\mathrm{W}$, Harrington $\mathrm{A}$, Wilson $\mathrm{C}$, Tsouros $\mathrm{S}$, Soobiah $\mathrm{C}$, Yu CH, Hutton B, Hoch JS, Hemmelgarn BR, Moher D, Majumdar SR, Straus SE (2014) Safety, effectiveness, and cost effectiveness of long acting versus intermediate acting insulin for patients with type 1 diabetes: systematic review and network meta-analysis. BMJ (Clin Res educ) 349:g5459. https://doi.org/10.1136/bmj. g5459

25. Chaimani A, Higgins JP, Mavridis D, Spyridonos P, Salanti G (2013) Graphical tools for network meta-analysis in STATA. PLoS One 8:e76654. https://doi.org/10.1371/journal.pone.0076654

26. Salanti G, Ades AE, Ioannidis JP (2011) Graphical methods and numerical summaries for presenting results from multiple-treatment meta-analysis: an overview and tutorial. J Clin Epidemiol 64:163-171. https://doi.org/10.1016/j.jclinepi.2010.03.016

27. Jansen JP, Naci H (2013) Is network meta-analysis as valid as standard pairwise meta-analysis? It all depends on the distribution of effect modifiers. BMC Med 11:159. https://doi. org/10.1186/1741-7015-11-159

28. Song F, Altman DG, Glenny AM, Deeks JJ (2003) Validity of indirect comparison for estimating efficacy of competing interventions: empirical evidence from published meta-analyses. BMJ 326:472. https://doi.org/10.1136/bmj.326.7387.472

29. Dias S, Welton NJ, Caldwell DM, Ades AE (2010) Checking consistency in mixed treatment comparison meta-analysis. Stat Med 29:932-944. https://doi.org/10.1002/sim.3767

30. White IR, Barrett JK, Jackson D, Higgins JP (2012) Consistency and inconsistency in network meta-analysis: model estimation using multivariate meta-regression. Res Synth Methods 3:111125. https://doi.org/10.1002/jrsm.1045

31. White IR (2011) Multivariate random-effects meta-regression: updates to mvmeta. Stata J 11:255-270

32. White IR (2015) Network meta-analysis. Stata J 15:951-985

33. Salanti G, Mavridis D, Nikolakopoulou A, Chaimani A, Efthimiou O (2018) Using Stata for standard pairwise and network meta-analysis. http://www.mtm.uoi.gr/index.php/stata-routinesfor-network-meta-analysis. Accessed 21 Sept 2018 
34. Chaimani A, Salanti G (2015) Visualizing assumptions and results in network meta-analysis: the network graphs package. Stata J 15:905-950

35. Bashir KMI, Choi JS (2017) Clinical and physiological perspectives of beta-glucans: the past, present, and future. Int J Mol Sci. https://doi.org/10.3390/ijms18091906

36. Vahouny GV, Tombes R, Cassidy MM, Kritchevsky D, Gallo LL (1980) Dietary fibers: V. Binding of bile salts, phospholipids and cholesterol from mixed micelles by bile acid sequestrants and dietary fibers. Lipids 15:1012-1018

37. Lia A, Hallmans G, Sandberg AS, Sundberg B, Aman P, Andersson $H$ (1995) Oat beta-glucan increases bile acid excretion and a fiber-rich barley fraction increases cholesterol excretion in ileostomy subjects. Am J Clin Nutr 62:1245-1251

38. Bodenlenz M, Ellmerer M, Schaupp L, Jacobsen LV, Plank J, Brunner GA, Wutte A, Aigner B, Mautner SI, Pieber TR (2015) Bioavailability of insulin detemir and human insulin at the level of peripheral interstitial fluid in humans, assessed by open-flow microperfusion. Diabetes Obes Metab 17:1166-1172. https://doi. org/10.1111/dom.12551

39. Kovatcheva-Datchary P, Nilsson A, Akrami R, Lee YS, De Vadder F, Arora T, Hallen A, Martens E, Bjorck I, Backhed F (2015) Dietary fiber-induced improvement in glucose metabolism is associated with increased abundance of prevotella. Cell Metab 22:971-982. https://doi.org/10.1016/j.cmet.2015.10.001

40. Martinez I, Lattimer JM, Hubach KL, Case JA, Yang J, Weber CG, Louk JA, Rose DJ, Kyureghian G, Peterson DA, Haub MD, Walter J (2013) Gut microbiome composition is linked to whole grain-induced immunological improvements. ISME J 7:269-280. https://doi.org/10.1038/ismej.2012.104

41. Vanegas SM, Meydani M, Barnett JB, Goldin B, Kane A, Rasmussen H, Brown C, Vangay P, Knights D, Jonnalagadda S, Koecher K, Karl JP, Thomas M, Dolnikowski G, Li L, Saltzman E, Wu D, Meydani SN (2017) Substituting whole grains for refined grains in a 6-wk randomized trial has a modest effect on gut microbiota and immune and inflammatory markers of healthy adults. Am J Clin Nutr 105:635-650. https://doi.org/10.3945/ajen.116.146928

42. Fardet A (2010) New hypotheses for the health-protective mechanisms of whole-grain cereals: what is beyond fibre? Nutr Res Rev 23:65-134. https://doi.org/10.1017/s0954422410000041

43. Cho SS, Qi L, Fahey GC Jr, Klurfeld DM (2013) Consumption of cereal fiber, mixtures of whole grains and bran, and whole grains and risk reduction in type 2 diabetes, obesity, and cardiovascular disease. Am J Clin Nutr 98:594-619. https://doi.org/10.3945/ ajen.113.067629 